\title{
FULL QUADRATURE SUMS FOR GENERALIZED POLYNOMIALS WITH FREUD WEIGHTS
}

\author{
HAEWON JOUNG
}

\begin{abstract}
Generalized nonnegative polynomials are defined as products of nonnegative polynomials raised to positive real powers. The generalized degree can be defined in a natural way. In this paper we extend quadrature sums involving $p$ th powers of polynomials to those for generalized polynomials.
\end{abstract}

\section{Introduction}

In 1969, Askey [1] proposed the following problem: Let $P \in \mathbb{P}_{n}$, where $\mathbb{P}_{n}$ denotes the set of all polynomials of degree at most $n$. Let $\left\{x_{n, j}\right\}$ be the zeros of the orthogonal polynomials with respect to $d \alpha$, a positive measure on $[-1,1]$, and $\left\{\lambda_{n, j}\right\}$ be the Cotes numbers for $d \alpha$. When is it true that

$$
\sum_{j=1}^{n} \lambda_{n, j}\left|P\left(x_{n, j}\right)\right|^{p} \leq C \int_{-1}^{1}|P(x)|^{p} d \alpha(x),
$$

where $C$ is independent of $P$ and $n$ ? Such inequalities are essential in various problems in approximation theory, and in particular, in investigating mean convergence of Lagrange interpolation and orthogonal expansions.

Of course when $p=2$, the Gauss quadrature formula asserts equality in (1.1) with $C=1$. Askey proved (1.1) for certain Jacobi weights for $p \geq 1$. Nevai [15] proved (1.1) for generalized Jacobi weights and $P \in \mathbb{P}_{l n}$ with $l \geq 2$ fixed. A further generalization, valid for $0<p<\infty$, and $P \in \mathbb{P}_{l n}$ with $l>1$ fixed, was proved in [10]. A converse inequality has been proven in [16].

For the Freud weights, Lubinsky, Máté, and Nevai [10, Corollary 9, p. 536] proved (1.1) with the range of summation suitably restricted, and subsequently, Lubinsky and Matjila [13] provided a solution for the Freud weights as follows: Let $r>0$, and $b \in(-\infty, 2]$. Then we have for $1 \leq p<\infty$

$$
\sum_{j=1}^{n} \lambda_{n, j}|P W|^{p}\left(x_{n, j}\right) W^{-b}\left(x_{n, j}\right) \leq C \int_{-\infty}^{\infty}|P W|(t) W^{2-b}(t) d t
$$

Received January 6, 2010; Revised January 15, 2010.

2000 Mathematics Subject Classification. 41A55.

Key words and phrases. quadrature sums, Freud weights, generalized polynomials. 
for every polynomial $P$ of degree at most $n+r n^{1 / 3}$, where $W^{2}(x)=\exp \left(-|x|^{\alpha}\right)$, $(\alpha>1),\left\{\lambda_{n, j}\right\}$ are the Cotes numbers, and $\left\{x_{n, j}\right\}$ are the zeros of the orthonormal polynomials for $W^{2}$.

The aim of this paper is to extend inequalities such as (1.2) to generalized polynomials.

A generalized nonnegative algebraic polynomial is a function of the type

$$
f(z)=|\omega| \prod_{j=1}^{m}\left|z-z_{j}\right|^{r_{j}} \quad(0 \neq \omega \in \mathbb{C})
$$

with $r_{j} \in \mathbb{R}^{+}, z_{j} \in \mathbb{C}$, and the number

$$
n \stackrel{\text { def }}{=} \sum_{j=1}^{m} r_{j}
$$

is called the generalized degree of $f$. Note that $n>0$ is not necessarily an integer. Thus throughout this paper we assume that $n \in \mathbb{R}^{+}$unless stated otherwise.

We denote by $\mathrm{GANP}_{n}$ the set of all generalized nonnegative algebraic polynomials of degree at most $n \in \mathbb{R}^{+}$.

Using

$$
\left|z-z_{j}\right|^{r_{j}}=\left(\left(z-z_{j}\right)\left(z-\bar{z}_{j}\right)\right)^{r_{j} / 2}, \quad z \in \mathbb{R},
$$

we can easily check that when $f \in \mathrm{GANP}_{n}$ is restricted to the real line, then it can be written as

$$
f=\prod_{j=1}^{m} P_{j}^{r_{j} / 2}, \quad 0 \leq P_{j} \in \mathbb{P}_{2}, \quad r_{j} \in \mathbb{R}^{+}, \quad \sum_{j=1}^{m} r_{j} \leq n,
$$

which is the product of nonnegative polynomials raised to positive real powers. This explains the name generalized nonnegative polynomials. Many properties of generalized nonnegative polynomials were investigated in a series of papers $([2,3,4,5])$.

Associated with the Freud weight $W_{\alpha}(x)=\exp \left(-|x|^{\alpha}\right), \alpha>0$, there are Mhaskar-Rahmanov-Saff numbers $a_{n}=a_{n}(\alpha)$, which is the positive solution of the equation

$$
n=\frac{2}{\pi} \int_{0}^{1} a_{n} t Q^{\prime}\left(a_{n} t\right)\left(1-t^{2}\right)^{-\frac{1}{2}} d t, \quad n \in \mathbb{R}^{+},
$$

where $Q(x)=|x|^{\alpha}, \alpha>0$. Explicitly,

$$
a_{n}=a_{n}(\alpha)=\left(\frac{n}{\lambda_{\alpha}}\right)^{1 / \alpha}, \quad n \in \mathbb{R}^{+},
$$

where

$$
\lambda_{\alpha}=\frac{2^{2-\alpha} \Gamma(\alpha)}{\{\Gamma(\alpha / 2)\}^{2}}
$$


Its importance lies partly in the identity

$$
\left\|P W_{\alpha}\right\|_{L^{\infty}(\mathbb{R})}=\left\|P W_{\alpha}\right\|_{L^{\infty}\left(\left[-a_{n}, a_{n}\right]\right)}, \quad P \in \mathbb{P}_{n} .
$$

Now we state our result.

Theorem 1.1. Let $W_{\alpha}(x)=\exp \left(-|x|^{\alpha}\right), \alpha>1$, and $0<p<\infty$. Let $K>0$, $\ell \geq 1$, and let

$$
-K a_{n} \leq y_{M}<y_{M-1}<\cdots<y_{1} \leq K a_{n}
$$

and

$$
\delta=\min \left\{y_{j-1}-y_{j}: j=2,3, \ldots, M\right\}>0 .
$$

Let $\Psi$ be convex, nonnegative, and nondecreasing in $[0, \infty)$. Then for all $f \in$ $\mathrm{GANP}_{\ell n}, \frac{2 p^{2}}{\ell p+4} \leq n \in \mathbb{R}^{+}$,

$$
\sum_{j=1}^{M} \Psi\left(f\left(y_{j}\right) W_{\alpha}^{p}\left(y_{j}\right)\right) \leq C_{1}\left(\frac{n}{a_{n}}+\frac{1}{\delta}\right) \int_{-\infty}^{\infty} \Psi\left(C_{2} f(u) W_{\alpha}^{p}(u)\right) d u .
$$

The constants $C_{1}$ and $C_{2}$ are independent of $M, \delta,\left\{y_{j}\right\}, n$, and $f$.

Theorem 1.2. Let $W_{\alpha}(x)=\exp \left(-|x|^{\alpha}\right), \alpha>1$. Let $0<p<\infty, \ell \geq 1$, and $\frac{2 p^{2}}{\ell p+4} \leq n \in \mathbb{N}$. Let $\left\{x_{n, j}\right\}$ be the zeros of orthogonal polynomial $P_{n}\left(W_{\alpha}^{2} ; x\right)$ and $\left\{\lambda_{n, j}\right\}$ be the Cotes numbers for $W_{\alpha}^{2}$. Then, there exists a positive constant $C$ such that

$$
\begin{aligned}
& \sum_{j=1}^{n} \lambda_{n, j} f\left(x_{n, j}\right) W_{\alpha}^{p-2}\left(x_{n, j}\right)\left(\max \left\{n^{-\frac{2}{3}}, 1-\frac{\left|x_{n, j}\right|}{a_{n}}\right\}\right)^{\frac{1}{2}} \\
\leq & C \int_{-\infty}^{\infty} f(u) W_{\alpha}^{p}(u) d u
\end{aligned}
$$

for $f \in \mathrm{GANP}_{\ell n}$.

In proving (1.2), refined Markov type inequalities [13] were used. We have to insert the square root factor on the left hand side of (1.4) because we do not have refined Markov type inequalities for generalized polynomials.

Throughout this paper we write $g_{n}(x) \sim h_{n}(x)$ if for every $n$ and for every $x$ in consideration

$$
0<c_{1} \leq \frac{g_{n}(x)}{h_{n}(x)} \leq c_{2}<\infty,
$$

and $g(x) \sim h(x), n \sim N$ have similar meanings.

\section{Proof of theorems}

In order to prove Theorems 1.1 and 1.2, we need lemmas on Infinite-Finite range inequalities and estimates of Christoffel functions for generalized polynomials.

In the analysis of extremal polynomials on $\mathbb{R}$, the estimation of the norm of a weighted polynomial $\|P W\|_{L^{p}(\mathbb{R})}$ in terms of the norm $\|P W\|_{L^{p}\left(-c_{n}, c_{n}\right)}$ over 
an finite interval $\left(-c_{n}, c_{n}\right)$ is important because such estimations or inequalities reduce problems over an infinite interval to problems on a finite interval. Mhaskar and Saff [14] established sharper inequalities that led to $n$th root asymptotics for extremal polynomials, for $p=\infty$ they showed that

$$
\left\|P W_{\alpha}\right\|_{L^{\infty}(\mathbb{R})}=\left\|P W_{\alpha}\right\|_{L^{\infty}\left(\left[-a_{n}, a_{n}\right]\right)}, \quad P \in \mathbb{P}_{n} .
$$

For generalized nonnegative polynomials we have the following lemma, which is the restatement of Theorem 2.1 in [7. p. 124].

Lemma 2.1. Let $W_{\alpha}(x)=\exp \left(-|x|^{\alpha}\right), \alpha>0$. Then

$$
\left\|f W_{\alpha}\right\|_{L^{\infty}(\mathbb{R})}=\left\|f W_{\alpha}\right\|_{L^{\infty}\left(\left[-a_{n}, a_{n}\right]\right)}
$$

for all $f \in \mathrm{GANP}_{n}, n \in \mathbb{R}^{+}$.

If $0<p<\infty$, then there exist positive constants $C_{1}$ and $C_{2}$ so that, whenever

$$
\frac{n}{(\log n)^{2}} \geq K_{n} \geq C_{1}, \quad 2 \leq n \in \mathbb{R}^{+}
$$

and

$$
\delta_{n}=\left(\frac{K_{n} \log n}{n}\right)^{2 / 3}, \quad 2 \leq n \in \mathbb{R}^{+}
$$

then

$$
\left\|f W_{\alpha}\right\|_{L^{p}(\mathbb{R})} \leq\left(1+n^{-C_{2} K_{n}}\right)\left\|f W_{\alpha}\right\|_{L^{p}\left(\left[-a_{n}\left(1+\delta_{n}\right), a_{n}\left(1+\delta_{n}\right)\right]\right)}
$$

for all $f \in \mathrm{GANP}_{n}, n \geq 2$.

Proof. See the proof of Theorem 2.1 in [7. p. 124].

Next we define generalized Christoffel functions. Let $0<p<\infty$. Then the generalized Christoffel function for ordinary polynomials is defined by

$$
\lambda_{n, p}\left(W_{\alpha} ; x\right)=\min _{P \in \mathbb{P}_{n-1}} \int_{-\infty}^{\infty} \frac{\left|P(t) W_{\alpha}(t)\right|^{p}}{|P(x)|^{p}} d t, \quad x \in \mathbb{R}, \quad n \in \mathbb{N} .
$$

The generalized Christoffel function for generalized nonnegative polynomials is defined by

$$
\omega_{n, p}\left(W_{\alpha} ; x\right)=\inf _{f \in \mathrm{GANP}_{n}} \int_{-\infty}^{\infty} \frac{\left(f(t) W_{\alpha}(t)\right)^{p}}{f^{p}(x)} d t, \quad x \in \mathbb{R}, \quad n \in \mathbb{R}^{+} .
$$

For the estimates of $\omega_{n, p}\left(W_{\alpha} ; x\right)$, we need the following lemma, which is the restatement of Theorem 2.3 in [7, p. 125].

Lemma 2.2. Let $W_{\alpha}(x)=\exp \left(-|x|^{\alpha}\right), \alpha>1$. Let $0<p<\infty$. Then

$$
\omega_{n, p}\left(W_{\alpha} ; x\right) \geq C \frac{a_{n}}{n} W_{\alpha}^{p}(x), \quad x \in \mathbb{R}, \quad n \in \mathbb{R}^{+},
$$

and

$$
\omega_{n, p}\left(W_{\alpha} ; x\right) \leq \lambda_{[n]+1, p}\left(W_{\alpha} ; x\right), \quad x \in \mathbb{R}, \quad n \in \mathbb{R}^{+},
$$

where $[n]$ denotes the integer part of $n$. 
Proof. See the proof of Theorem 2.3 in [7, p. 125].

Remark. It is well known (see, for example, [8]) that if $\alpha>1$, then there exist positive constants $C_{1}$ and $C_{2}$ depending on $p$ and $\alpha$, such that

$$
\lambda_{[n]+1, p}\left(W_{\alpha} ; x\right) \leq C_{1} \frac{a_{n}}{n} W_{\alpha}^{p}(x), \quad|x| \leq C_{2} a_{n} .
$$

Consequently

$$
\omega_{n, p}\left(W_{\alpha} ; x\right) \sim \frac{a_{n}}{n} W_{\alpha}^{p}(x), \quad|x| \leq C_{2} a_{n} .
$$

Now we prove our results.

Proof of Theorem 1.1. Let $W_{\alpha}(x)=\exp \left(-|x|^{\alpha}\right), \alpha>1,0<p<\infty$. Fix $K>0$ and $\ell \geq 1$. Let $\Psi$ be convex, nonnegative, and nondecreasing in $[0, \infty)$. By Lemma 2.1, there exists a positive constant $B^{*}$ such that

$$
\left\|f W_{\alpha}\right\|_{L^{p}(\mathbb{R})} \leq 2\left\|f W_{\alpha}\right\|_{L^{p}\left(\left[-B^{*} a_{n}, B^{*} a_{n}\right]\right)}, \quad(0<p<\infty)
$$

for $n \geq 2, f \in \operatorname{GANP}_{n}$. Let $k=4 / p$ and $B \geq B^{*}(\ell+k)$. Then

$$
B^{*} a_{(\ell+k) n} \leq B a_{n} .
$$

Now let $p_{j}(v, x), j=0,1,2, \ldots$, be the orthonormal Chebyshev polynomials associated with the Chebyshev weight

$$
v(t)=\left\{\begin{aligned}
\left(1-t^{2}\right)^{-1 / 2}, & t \in(-1,1), \\
0, & t \notin(-1,1) .
\end{aligned}\right.
$$

and let

$$
K_{m}(v, x, t)=\sum_{j=0}^{m-1} p_{j}(v, x) p_{j}(v, t), \quad m \in \mathbb{N} .
$$

Let $f \in \mathrm{GANP}_{\ell n},(\ell+k) n \geq 2$, and let $N=[n]+1$. Then for each fixed $x$,

$$
f(t)\left|K_{N}\left(v, \frac{x}{B a_{n}}, \frac{t}{B a_{n}}\right)\right|^{k}
$$

is a generalized polynomial in $t$ of degree less than $(\ell+k) n$. By Lemma 2.2, (2.5), and (2.6), we have for all $t \in \mathbb{R}$,

$$
\begin{aligned}
& f^{p}(t) W_{\alpha}^{p}(t)\left|K_{N}^{k}\left(v, x /\left(B a_{n}\right), t /\left(B a_{n}\right)\right)\right|^{p} \\
\leq & c_{1} \frac{n}{a_{n}} \int_{-\infty}^{\infty} f^{p}(u) W_{\alpha}^{p}(u) K_{N}^{4}\left(v, x /\left(B a_{n}\right), u /\left(B a_{n}\right)\right) d u \\
\leq & c_{2} \frac{n}{a_{n}} \int_{-B a_{n}}^{B a_{n}} f^{p}(u) W_{\alpha}^{p}(u) K_{N}^{4}\left(v, x /\left(B a_{n}\right), u /\left(B a_{n}\right)\right) d u
\end{aligned}
$$

Set $t=x$. Then for all $x \in \mathbb{R}$,

$$
\begin{aligned}
& f^{p}(x) W_{\alpha}^{p}(x) K_{N}^{4}\left(v, x /\left(B a_{n}\right), x /\left(B a_{n}\right)\right) \\
\leq & c_{2} \frac{n}{a_{n}} \int_{-B a_{n}}^{B a_{n}} f^{p}(u) W_{\alpha}^{p}(u) K_{N}^{4}\left(v, x /\left(B a_{n}\right), u /\left(B a_{n}\right)\right) d u .
\end{aligned}
$$


Since

$$
K_{N}^{4}\left(v, x /\left(B a_{n}\right), x /\left(B a_{n}\right)\right) \sim N^{4} \sim n^{4} \quad \text { for } \quad|x| \leq B a_{n},
$$

(see, [15, p. 108]), we have

(2.7) $f^{p}(x) W_{\alpha}^{p}(x) \leq c_{3} \frac{1}{n^{3} a_{n}} \int_{-B a_{n}}^{B a_{n}} f^{p}(u) W_{\alpha}^{p}(u) K_{N}^{4}\left(v, x /\left(B a_{n}\right), u /\left(B a_{n}\right)\right) d u$ for $|x| \leq B a_{n}$. By Theorem 2.2 in [10, p. 537], we have for $|x| \leq \frac{B a_{n}}{2}$,

$$
\begin{aligned}
& \int_{-b a_{n}}^{B a_{n}} K_{N}^{4}\left(v, x /\left(B a_{n}\right), u /\left(B a_{n}\right)\right) d u \\
= & B a_{n} \int_{-1}^{1} K_{N}^{4}\left(v, x /\left(B a_{n}\right), u\right) d u \sim a_{n} N^{3} \sim a_{n} n^{3} .
\end{aligned}
$$

Using Jensen's inequality and (2.7), we obtain for $|x| \leq \frac{B a_{n}}{2}$,

$$
\begin{aligned}
& \Psi\left(f^{p}(x) W_{\alpha}^{p}(x)\right) \\
\leq & \Psi\left(\frac{\int_{-B a_{n}}^{B a_{n}} c_{4} f^{p}(u) W_{\alpha}^{p}(u) K_{N}^{4}\left(v, x /\left(B a_{n}\right), u /\left(B a_{n}\right)\right) d u}{\int_{-B a_{n}}^{B a_{n}} K_{N}^{4}\left(v, x /\left(B a_{n}\right), u /\left(B a_{n}\right)\right) d u}\right) \\
\leq & \frac{\int_{-B a_{n}}^{B a_{n}} \Psi\left(c_{4} f^{p}(u) W_{\alpha}^{p}(u)\right) K_{N}^{4}\left(v, x /\left(B a_{n}\right), u /\left(B a_{n}\right)\right) d u}{\int_{-B a_{n}}^{B a_{n}} K_{N}^{4}\left(v, x /\left(B a_{n}\right), u /\left(B a_{n}\right)\right) d u} \\
\leq & c_{5} \frac{1}{a_{n} n^{3}} \int_{-B a_{n}}^{B a_{n}} \Psi\left(c_{4} f^{p}(u) W_{\alpha}^{p}(u)\right) K_{N}^{4}\left(v, x /\left(B a_{n}\right), u /\left(B a_{n}\right)\right) d u .
\end{aligned}
$$

Since

$$
K_{N}^{4}\left(v, \frac{x}{B a_{n}}, \frac{u}{B a_{n}}\right) \leq c_{6} n^{2} K_{N}^{2}\left(v, \frac{x}{B a_{n}}, \frac{u}{B a_{n}}\right), \quad|x| \leq B a_{n}, \quad|u| \leq B a_{n}
$$

we have

$$
\begin{aligned}
& \Phi\left(f^{p}(x) W_{\alpha}^{p}(x)\right) \\
\leq & c_{7} \frac{1}{a_{n} n} \int_{-B a_{n}}^{B a_{n}} \Psi\left(c_{4} f^{p}(u) W_{\alpha}^{p}(u)\right) K_{N}^{2}\left(v, x /\left(B a_{n}\right), u /\left(B a_{n}\right)\right) d u
\end{aligned}
$$

for $|x| \leq \frac{B a_{n}}{2}$.

Now, let

$$
-K a_{n} \leq y_{M}<y_{M-1}<\cdots<y_{1} \leq K a_{n},
$$

and

$$
\delta=\min \left\{y_{j-1}-y_{i}: j=2,3, \ldots, M\right\}>0 .
$$

We can assume that $K \leq B / 2$ so that $\left|y_{j} /\left(B a_{n}\right)\right| \leq 1 / 2$ for $j=1,2, \ldots, M$. As

$$
\frac{d}{d x} \arccos (x) \sim-1 \quad \text { for } \quad|x| \leq 1 / 2
$$


we have

$$
\arccos \left(y_{j} /\left(B a_{n}\right)\right)-\arccos \left(y_{j-1} /\left(B a_{n}\right)\right) \geq c_{8} \frac{y_{j-1}-y_{j}}{a_{n}} \geq c_{8} \frac{\delta}{a_{n}} .
$$

Then by Lemma 2.3 in [10, p. 539], we obtain

$$
\sum_{j=1}^{M} K_{N}^{2}\left(v, \frac{y_{j}}{B a_{n}}, \frac{u}{B a_{n}}\right) \leq \frac{8}{\pi^{2}} N\left(N+\frac{c_{9} a_{n}}{\delta}\right) \quad \text { for } \quad|u| \leq B a_{n} .
$$

Using (2.8) and the above inequality, we have for all $f \in \operatorname{GANP}_{\ell n}, n \geq \frac{2}{\ell+k}=$ $\frac{2 p}{\ell p+4}$

$$
\sum_{j=1}^{M} \Psi\left(f^{p}\left(y_{j}\right) W_{\alpha}^{p}\left(y_{j}\right)\right) \leq c_{10}\left(\frac{n}{a_{n}}+\frac{1}{\delta}\right) \int_{-\infty}^{\infty} \Psi\left(c_{4} f^{p}(u) W_{\alpha}^{p}(u)\right) d u,
$$

which yields Theorem 1.1.

Lemma 2.3. Let $x_{n, n}<x_{n, n-1}<\cdots<x_{n, 1}$ be the zeros of orthogonal polynomial $P_{n}\left(W_{\alpha}^{2} ; x\right), \alpha>1$. Then there exists a positive constant $C$ such that

$$
x_{n, j-1}-x_{n, j} \geq C \frac{a_{n}}{n} \quad \text { for } \quad j=2, \ldots, n .
$$

Proof. By Theorem 5.1 in $[6$, p. 36], there exists $\eta \in(0,1)$ such that

$$
x_{n, j-1}-x_{n, j} \sim \frac{a_{n}}{n} \text { for }\left|x_{n, j}\right| \leq \eta a_{n} .
$$

By Theorem 7.6 in $[8$, p. 168]

$$
\left(\int_{-\infty}^{\infty}\left|P(t) W_{\alpha}(t)\right|^{2} d t\right)^{\frac{1}{2}} \geq c_{1}\left(\frac{a_{n}}{n}\right)^{\frac{1}{2}}\left\|P W_{\alpha}\right\|_{L^{\infty}(\mathbb{R})}, \quad P \in \mathbb{P}_{n-1} .
$$

Let

$$
g_{n}(x)=\left(\max \left\{n^{-\frac{2}{3}}, 1-\frac{|x|}{a_{n}}\right\}\right)^{\frac{1}{2}} .
$$

By Theorem 1.9 in [9, p. 470],

$$
\left\|P W_{\alpha}\right\|_{L^{\infty}(\mathbb{R})} \geq c_{2} \frac{a_{n}}{n} g_{n}^{-1}(x)\left|\left(P W_{\alpha}\right)^{\prime}(x)\right|
$$

for $|x| \geq \eta a_{n}, P \in \mathbb{P}_{n-1}$. By (2.10) and (2.11), we have

$$
\frac{\int_{-\infty}^{\infty}\left|P(t) W_{\alpha}(t)\right|^{2} d t}{\mid\left(P W_{\alpha}\right)^{\prime 2}} \geq c_{3}\left(\frac{a_{n}}{n}\right)^{3} g_{n}^{-2}(x)
$$

for $|x| \geq \eta a_{n}$, and for all $P \in \mathbb{P}_{n-1}$, hence

$$
\inf _{P \in \mathbb{P}_{n-1}} \frac{\int_{-\infty}^{\infty}\left|P(t) W_{\alpha}(t)\right|^{2} d t}{\mid\left(P W_{\alpha}\right)^{\prime 2}} \geq c_{3}\left(\frac{a_{n}}{n}\right)^{3} g_{n}^{-2}(x) \quad \text { for } \quad|x| \geq \eta a_{n} .
$$


Since

$$
\inf _{P \in \mathbb{P}_{n-1}} \frac{\int_{-\infty}^{\infty}\left|P(t) W_{\alpha}(t)\right|^{2} d t}{\mid\left(P W_{\alpha}\right)^{\prime 2}}=\frac{1}{\sum_{i=0}^{n-1}\left(\frac{d}{d x} P_{i}\left(W_{\alpha}^{2} ; x\right) W_{\alpha}(x)\right)^{2}},
$$

(see, [6, Lemma 2.1, p. 24]), we have

$$
\sum_{i=0}^{n-1}\left(\frac{d}{d x} P_{i}\left(W_{\alpha}^{2} ; x\right) W_{\alpha}(x)\right)^{2} \leq c_{4}\left(\frac{n}{a_{n}}\right)^{3} g_{n}^{2}(x), \quad|x| \geq \eta a_{n} .
$$

Now let

$$
K_{n}\left(W_{\alpha}^{2} ; t, x\right)=\sum_{i=0}^{n-1} P_{i}\left(W_{\alpha}^{2} ; t\right) P_{i}\left(W_{\alpha}^{2} ; x\right) .
$$

Then by the Christofell-Darboux formula, we have

$$
K_{n}\left(W_{\alpha}^{2} ; x_{n, j}, x\right)=\frac{\gamma_{n-1}\left(W_{\alpha}^{2}\right)}{\gamma_{n}\left(W_{\alpha}^{2}\right)} \frac{P_{n-1}\left(W_{\alpha}^{2} ; x_{n, j}\right) P_{n}\left(W_{\alpha}^{2} ; x\right)}{x-x_{n, j}},
$$

which implies that

$$
K_{n}\left(W_{\alpha}^{2} ; x_{n, j}, x_{n, j+1}\right)=0 .
$$

By Theorem 1.1 in [9, p. 465] and Corollary 1.2 in [9, p. 466], we have

$$
\sum_{i=0}^{n-1} P_{i}^{2}\left(W_{\alpha}^{2} ; x_{n, j}\right) \sim \frac{n}{a_{n}} W_{\alpha}^{-2}\left(x_{n, j}\right) g_{n}\left(x_{n, j}\right)
$$

for all $n=1,2, \ldots$, and for $j=1,2, \ldots, n$.

Now suppose that $x_{n, j} \geq x \geq x_{n, j+1} \geq \eta a_{n}$. Then

$$
g_{n}\left(x_{n, j}\right) \leq g_{n}(x) \leq g_{n}\left(x_{n, j+1}\right) .
$$

By (2.12), (2.14), and (2.15), we have

$$
\begin{aligned}
& \left|\frac{d}{d x} K_{n}\left(W_{\alpha}^{2} ; x_{n, j}, x\right) W_{\alpha}(x)\right| \\
= & \left|\sum_{i=0}^{n-1} P_{i}\left(W_{\alpha}^{2} ; x_{n, j}\right) \frac{d}{d x}\left(P_{i}\left(W_{\alpha}^{2} ; x\right) W_{\alpha}(x)\right)\right| \\
\leq & \left(\sum_{i=0}^{n-1} P_{i}^{2}\left(W_{\alpha}^{2} ; x_{n, j}\right) \sum_{i=0}^{n-1}\left(\frac{d}{d x}\left(P_{i}\left(W_{\alpha}^{2} ; x\right) W_{\alpha}(x)\right)\right)^{2}\right)^{1 / 2} \\
\leq & c_{5}\left(\left(\frac{n}{a_{n}}\right)^{4} W_{\alpha}^{-2}\left(x_{n, j}\right) g_{n}\left(x_{n, j}\right) g_{n}^{2}(x)\right)^{1 / 2} \\
\leq & c_{5}\left(\frac{n}{a_{n}}\right)^{2} W_{\alpha}^{-1}\left(x_{n, j}\right) g_{n}^{\frac{3}{2}}\left(x_{n, j+1}\right) .
\end{aligned}
$$


From (2.13), (2.14), and (2.16), we have

$$
\begin{aligned}
& c_{6} \frac{n}{a_{n}} g_{n}\left(x_{n, j}\right) W_{\alpha}^{-1}\left(x_{n, j}\right) \\
\leq & K_{n}\left(W_{\alpha}^{2} ; x_{n, j}, x_{n, j}\right) W_{\alpha}\left(x_{n, j}\right) \\
= & K_{n}\left(W_{\alpha}^{2} ; x_{n, j}, x_{n, j}\right) W_{\alpha}\left(x_{n, j}\right)-K_{n}\left(W_{\alpha}^{2} ; x_{n, j}, x_{n, j+1}\right) W_{\alpha}\left(x_{n, j+1}\right) \\
= & \int_{x_{n, j+1}}^{x_{n, j}} \frac{d}{d x}\left(K_{n}\left(W_{\alpha}^{2} ; x_{n, j}, x\right) W_{\alpha}(x)\right) d x \\
\leq & c_{7}\left(\frac{n}{a_{n}}\right)^{2} W_{\alpha}^{-1}\left(x_{n, j}\right) g_{n}^{\frac{3}{2}}\left(x_{n, j+1}\right)\left(x_{n, j}-x_{n, j+1}\right) .
\end{aligned}
$$

Since $g_{n}\left(x_{n, j}\right) \sim g_{n}\left(x_{n, j+1}\right)$, (see, [9, (11.10), p. 521]), from (2.17), we have for $x_{n, j}>x_{n, j+1} \geq \eta a_{n}$,

$$
x_{n, j}-x_{n, j+1} \geq c_{8} \frac{a_{n}}{n} g_{n}^{-\frac{1}{2}}\left(x_{n, j+1}\right) \geq c_{9} \frac{a_{n}}{n} .
$$

The proof of (2.18) for $x_{n, j+1}<x_{n, j} \leq-\eta a_{n}$, is similar, hence by (2.8) and (2.18), Lemma 2.3 follows.

Proof of Theorem 1.2. Let $0<p<\infty$ and let $n \in \mathbb{N}$. Let $\left\{x_{n, j}\right\}$ be the zeros of orthogonal polynomial $P_{n}\left(W_{\alpha}^{2} ; x\right)$ and $\left\{\lambda_{n, j}\right\}$ be the Cotes numbers for $W_{\alpha}^{2}$. By Theorem 1.1 in [9, p. 465] and Corollary 1.2 in [9, p. 466], we have for all $n \in \mathbb{N}$, and $j=1,2, \ldots, n$,

$$
\lambda_{n, j} \leq c \frac{a_{n}}{n}\left(\max \left\{n^{-\frac{2}{3}}, 1-\frac{\left|x_{n, j}\right|}{a_{n}}\right\}\right)^{-1 / 2} W_{\alpha}^{2}\left(x_{n, j}\right) .
$$

Let $f \in \operatorname{GANP}_{\ell n}$. Then by (2.19), we have for all $j=1,2, \ldots, n$,

$$
\begin{aligned}
& \lambda_{n, j} f\left(x_{n, j}\right) W_{\alpha}^{p-2}\left(x_{n, j}\right) \quad\left(\max \left\{n^{-\frac{2}{3}}, 1-\frac{\left|x_{n, j}\right|}{a_{n}}\right\}\right)^{1 / 2} \\
\leq & c \frac{a_{n}}{n} f\left(x_{n, j}\right) W_{\alpha}^{p}\left(x_{n, j}\right),
\end{aligned}
$$

hence

$$
\begin{aligned}
& \sum_{j=1}^{n} \lambda_{n, j} f\left(x_{n, j}\right) W_{\alpha}^{p-2}\left(x_{n, j}\right)\left(\max \left\{n^{-\frac{2}{3}}, 1-\frac{\left|x_{n, j}\right|}{a_{n}}\right\}\right)^{1 / 2} \\
\leq & c \frac{a_{n}}{n} \sum_{j=1}^{n} f\left(x_{n, j}\right) W_{\alpha}^{p}\left(x_{n, j}\right) .
\end{aligned}
$$

Using Theorem 1.1 with $\Psi(x)=x$, we have

$$
\sum_{j=1}^{n} f\left(x_{n, j}\right) W_{\alpha}^{p}\left(x_{n, j}\right) \leq c_{1}\left(\frac{n}{a_{n}}+\frac{1}{\delta}\right) \int_{-\infty}^{\infty} f(u) W_{\alpha}^{p}(u) d u
$$

where

$$
\delta=\min \left\{x_{n, j}-x_{n, j-1}: j=2,3, \ldots, n\right\}>0 .
$$


By Lemma 2.3,

thus

$$
\frac{1}{\delta} \leq c_{2} \frac{n}{a_{n}}
$$

$$
\sum_{j=1}^{n} f\left(x_{n, j}\right) W_{\alpha}^{p}\left(x_{n, j}\right) \leq c_{3} \frac{n}{a_{n}} \int_{-\infty}^{\infty} f(u) W_{\alpha}^{p}(u) d u .
$$

Then Theorem 1.2 follows from (2.20) and (2.21).

Acknowledgments. The author thanks P. Nevai for his helpful suggestions.

\section{References}

[1] R. Askey, R. DeVore, G. Freud, J. Musielak, J. Peetre, and T. Popoviciu, Proposed problems, Proceedings of the Conference on the Constructive Theory of Functions (Approximation Theory) (Budapest, 1969), pp. 533-538. Akademiai Kiado, Budapest, 1972.

[2] T. Erdélyi, Bernstein and Markov type inequalities for generalized nonnegative polynomials, Canad. J. Math. 43 (1991), no. 3, 495-505.

[3] __ Remez-type inequalities on the size of generalized polynomials, J. London Math. Soc. (2) 45 (1992), no. 2, 255-264.

[4] T. Erdélyi, A. Máté, and P. Nevai, Inequalities for generalized nonnegative polynomials, Constr. Approx. 8 (1992), no. 2, 241-255.

[5] T. Erdélyi and P. Nevai, Generalized Jacobi weights, Christoffel functions, and zeros of orthogonal polynomials, J. Approx. Theory 69 (1992), no. 2, 111-132.

[6] G. Freud, On Markov-Bernstein-type inequalities and their applications, J. Approximation Theory 19 (1977), no. 1, 22-37.

[7] H. Joung, Estimates of Christoffel functions for generalized polynomials with exponential weights, Commun. Korean Math. Soc. 14 (1999), no. 1, 121-134.

[8] A. L. Levin and D. S. Lubinsky, Canonical products and the weights $\exp \left(-|x|^{\alpha}\right), \alpha>1$, with applications, J. Approx. Theory 49 (1987), no. 2, 149-169.

[9] _ Christoffel functions, orthogonal polynomials, and Nevai's conjecture for Freud weights, Constr. Approx. 8 (1992), no. 4, 463-535.

[10] D. S. Lubinsky, A. Máté, and P. Nevai, Quadrature sums involving pth powers of polynomials, SIAM J. Math. Anal. 18 (1987), no. 2, 531-544.

[11] D. S. Lubinsky, H. N. Mhaskar, and E. B. Saff, A proof of Freud's conjecture for exponential weights, Constr. Approx. 4 (1988), no. 1, 65-83.

[12] D. S. Lubinsky and E. B. Saff, Strong Asymptotics for Extremal Polynomials Associated with Weights on R, Lecture Notes in Mathematics, 1305. Springer-Verlag, Berlin, 1988.

[13] D. S. Lubinsky and D. M. Matjila, Full quadrature sums for pth powers of polynomials with Freud weights, J. Comput. Appl. Math. 60 (1995), no. 3, 285-296.

[14] H. N. Mhaskar and E. B. Saff, Extremal problems for polynomials with exponential weights, Trans. Amer. Math. Soc. 285 (1984), no. 1, 203-234.

[15] P. Nevai, Orthogonal polynomials, Mem. Amer. Math. Soc. 18 (1979), no. 213, v+185 pp.

[16] Y. Xu, Mean convergence of generalized Jacobi series and interpolating polynomials. II, J. Approx. Theory 76 (1994), no. 1, 77-92.

DEPARTMENT OF MATHEMATICS

INHA UNIVERSITY

INCHEON 402-751, KOREA

E-mail address: hwjoung@inha.ac.kr 